\title{
School-based Learning for Individual Diversity in Education: The SLIDE Project
}

\author{
Ingrid Harrington \\ School of Education, University of New England, Australia \\ Correspondence: Ingrid Harrington, School of Education, University of New England, Australia. Tel: \\ 61-2-6773-5068. E-mail: iharring@une.edu.au
}

Received: March 26, 2013 Accepted: April 7, 2013 Available online: May 3, 2013

doi:10.11114/jets.v1i2.112 URL: http://dx.doi.org/10.11114/jets.v1i2.112

\begin{abstract}
Whole-School Professional Development (PD) days held regularly in schools are designed to promote and up-skill school staff on relevant areas of educational policy and practice. An aim of PD days is to empower participants with new knowledges and skills, and to confirm that current practices and interpretations of education policy and procedure are correct. In determining the nature of the professional development content, school executives make decisions based on their perceived needs of the staffing group, available speakers, current departmental policy and practice requirements. Deciding the topics for the professional development day for school staff has tended to adopt a 'one size fits all' general approach that is rationalised as being broad in nature and of benefit to all. In adopting such an approach to a diverse teaching cohort, one may question just how valuable and meaningful this training day is in meeting the individual needs of all participating teachers.

This paper reports on the benefits a more individualised approach to a whole-school PD training day had for one government primary school in Australia. Foundational to the detailed design of the PD days, the 'School-based Learning for Individual Diversity in Education' (SLIDE) project proceeded by identifying the individual needs of each staff member in the school community through an interview prior to the delivery of the two-day training. Embedded in an action research model, the SLIDE project reported evidence of improved teacher self-confidence, improved communication between all levels, and a boost to the level of whole-school staff cohesion and morale.
\end{abstract}

Keywords: teacher professional development, teacher training

\section{Introduction}

Schools need to recognise and accommodate the differing professional needs inherent with the broad diversity in the varied roles of administrative, support and teaching staff that synergise the learning culture and school community (Kedzior \& Fifield, 2004; Van Driel \& Berry, 2012). This paper argues that when considering the whole-school professional development of a school's learning community of staff, a "one size fits all" approach may not be the most effective in identifying, addressing, and meeting each individual's learning needs. This research borrows from the Pedagogy, Content, Knowledge (PCK) model (Anderson \& Herr, 2011; Van Driel \& Berry, 2012) and embeds the seven most consistently cited factors by teachers into a two-day whole-school professional development series to improve the overall confidence, understanding, communication and professional practice of the school's learning community. Of significance is the time taken to individually interview each member of the learning community prior to the training, and then use this data as the foundation of the two-day training program for staff.

\section{Literature Review}

It has been acknowledged that the professional development of teachers spans their entire career (Bielaczyc \& Collins, 1999; Borko, Jacobs, \& Koellner, 2010; Gutsky, 2003). Teaching success in classrooms is promoted through professional development sessions that consider content and knowledge skills that underpin instructional and curriculum decisions (Opfer \& Pedder, 2011). The forms professional development can take are numerous and varied including: continuing education, in-service and pre-service education; group work, individual, peer support and collaboration. However, research by Rhoton \& Stile, $(2002$, p.1) reported that generalised "teacher 
development as carried out in most schools today is not designed to develop the teacher expertise needed to bring about improved student learning".

There is a growing research base which advocates that effective teacher professional development needs to have a strong focus on Pedagogy, Content, Knowledge (PCK) that encompasses the teacher's understanding of student learning and subject content (Anderson \& Herr, 2011; Van Driel \& Berry, 2012). In this instance, teachers actively engage in critical reflection of their own professional practice. This focus deviates from platforms of professional development where models of 'expert teaching' are promoted as an ideal or a 'best' practice way of managing and teaching students is advocated (Bausmith \& Barry, 2011; Browder, et al, 2012), without an integration into schema through critical reflection.

The concept of a 'learning community' (Wenger, 1998) is a concept applied to both student and staff cohorts in schools. Vrasidas and Glass (2004) recognised that students learn best when they are intrinsically motivated; work on challenging, relevant and authentic activities; collaborate with, give and receive feedback, to their peers; exchange ideas; engage in critical reflection. The same learning conditions can be applied to teachers. Teachers state they respond best to professional development include training that: is 'extended' i.e. not a one-off session; involves collaboration with peers; has ongoing support with follow-up; is coherent and integrated; inquiry-based i.e. promotes inquiry and reflection through active learning; is teacher-driven i.e. addresses individual and/or organisational needs; and provides the opportunity for self-evaluation (Borko, Jacobs, \& Koellner, 2010; Kedzior \& Fifield, 2004; Little, 2006; Van Driel \& Berry, 2012). The design of the SLIDE training program ensured that these seven factors remained paramount. Incorporating the individual data from each member of the school community ensured that the training would be relevant, of interest and meet the professional and/or personal training needs they identified.

Vrasidas and Glass (2004) suggest that when everyone is involved in a collective effort of understanding, the learning culture itself defines the quality of the learning community. Bielaczyc and Collins (1999) claim that effective learning cultures comprise four characteristics: i) a diversity of expertise amongst its members that is valued and supported; ii) a shared objective of continually advancing collective knowledge and skills; iii) an emphasis on how to learn best; and, iv) mechanisms for sharing what is learned. The issue for the school referred to in this paper was that its learning culture and therefore learning community, were incoherent and dysfunctional due to a number of factors. Whilst there exist a diversity of expertise amongst its members, this was not publicly valued and supported. As such, there was no shared objective of continually advancing the collective knowledge and skills, the emphasis on how to learn best was lost, and there were no mechanisms for sharing information (Bielaczyc \& Collins 1999).

The purpose of the SLIDE training was to try and improve the learning culture that would benefit the quality of the learning community. In discussion with the school executive, the perception was that staff did not respect nor listen to each other and after a while, vital communication ceased all together in the school community. Resonating from Friere's (1970, p. 54) belief that "if the structure does not permit dialogue the structure must be changed", the facilitator acknowledged and listened to the individual staff voice, an approach not previously taken. The facilitator proceeded by providing a safe, confidential and private 'space' where each member of the school community could express themselves without fear of bias, judgement or repercussion.

\section{Methodology}

The design, implementation and evaluation of the SLIDE program was in response to the relevant Department of Education's need to address falling staff morale and performance issues at one of their government primary schools. The school had experienced a high turnover of staff at the executive and teaching levels over the previous twelve months, and the levels of effective staff communication between teachers and the support staff formally known as Teacher Aides - appeared to be random, lack coherence and structure. This breakdown of communication promoted an environment of confusion and guesswork, and fuelled actions underpinned by a discourse of uncertainty, endorsing divisions amongst and between all staff at the school.

The entire school community - executive, teaching, administrative and support - participated in the SLIDE project interviews. The project recognised each participant's needs at an individual level aiming to provide staff with a space where they could privately voice professional/personal issues of concern, identify areas of efficiency, and make suggestions on how the school could improve its overall effectiveness to staff and students. The SLIDE facilitator was invited to present the training details at one of the weekly staff meetings. The exact nature and purpose of the PD sessions was outlined as was the lead-up process to the training days: the purpose and scheduling of the individual interviews; assurance of confidentiality and privacy; and clarification of the role 
and expectations of the facilitator and participant. Information sheets and consent forms were distributed to all participants.

Over a period of two weeks each staff member participated in a 1:1 taped semi-structured interview with the researcher for approximately 30-40 minutes. The nature of the questions was deliberately open-ended allowing staff to fully elaborate and speak freely about their experiences, and minimising research control over their definitions and expression of their experiences (Renwick et al., 2003). The questions required staff to reflect and identify current efficiencies and deficiencies in their workplace, self-assess their knowledge-base of the department's policies and procedures, and to discuss their current levels of professional self-esteem and confidence in the workplace. The personal interviews provided the opportunity for the researcher to pool each response to gauge a) the nature of school-based issues raised as a concern, b) current practices that were deemed to be effective, c) the training needs identified by teachers, support staff and administrative staff, d) any similarities and differences of those training needs between the participants, and e) a 'whole-school' perspective of training gaps and needs. The facilitator was mindful to avoid inadvertently perpetuating any school-based negativity so when inviting staff to share concerns, they were required to put forward suggestions on how they would address the identified inefficiency. Central to the success of the project and to preserve its objective focus, all participants were assured by the researcher that the 1:1 interviews would remain private, the details of the conversations confidential, and their identity remain anonymous from other participants and the school executives.

The PD training was scheduled over two sessions: the first session was held after school for two hours, and the second session was held for one hour six weeks later. The purpose of the first session was for the researcher to report collectively and anonymously the identified needs from the staff interviews, and to address these needs through the clarification of policy, practice, provision of a resource folder, and suggestions of future in-school procedures. The second session held six weeks later provided staff with an opportunity to report on the success of a number of strategies and initiatives discussed in the first session. Underpinning the second session was the concept that staff needed to be active agents for their own learning and make positive changes to affect others, rather than engage in a silent acceptance of ineffective processes and accept a lack of knowledge and understanding. The facilitator required the participants to be accountable and responsible for their own learning by asking what efforts they had made to implement or adopt new strategies to address their identified training need(s) from the first session. Through self-assessment, reflection as a group and individually, the participants were asked to gauge their overall satisfaction with any changes they had implemented during this time.

Analysis of the interview data using Friere's (1970) model of generative themes, revealed that three themes surrounding i) Communication, ii) Policy, and iii) Practice emerged. During the first session, the researcher presented the three data themes and instructional material as a Powerpoint presentation. Each participant received a resource folder that contained copies of department's policies, behaviour disorders, and response strategies that had been identified by the participants as areas needing clarification and attention. A self-assessment survey was designed around these three themes and distributed to all staff at the first PD session. The purpose of this survey was to further refine the interview data, and provide staff with an opportunity to self-assess their levels of professional and personal efficacy in the workplace, their current understandings of a range of the department's policies, and reflect on the effectiveness of their own work practices in areas relevant to their role through an action research model. The same survey was again distributed at the beginning of the second session to assess how effective the action research 'information transfer to practice' process had been.

The paper proceeds with a brief exploration of the three dominant themes that emerged from the staff interviews:

i) Communication, ii) Policy, and iii) Practice, and then discusses staff personal and professional growth post-training.

\section{Results}

\subsection{Communication}

All staff agreed that the communication between and amongst themselves was in need of improvement. When asked to explain why the level of communication was so poor, a teacher commented, "We are all just so busy. We don't have the time to stop and talk about things to each other" (Teacher 2). One staff member understood the lack of communication was the result of staff not placing colleague communication as a priority in the busy school day:

No-one talks to anybody around here! I find things out about kids in my class from the wrong people at the wrong time, all the time! It seems so stupid because we should all be on the same team, you know, 
helping the kids and all, but it doesn't seem to work like that. Teachers don't keep us in the loop with what we need to know, so most of the time we are flying blind (Support staff 2).

Despite staff acknowledging their regular attendance at the weekly staff meeting chaired by the Principal, they viewed this time as being "told what to do", rather than a time where everyone could "share what we are doing". Both teachers and support staff were vocal on how the poor levels of communication negatively affected their understanding of each other's roles and teaching. A number of support staff felt they had no real authority from the eyes of the student due to the teacher undermining their work with students. The teachers and support staff recognised that there was a lack of clarity around their job boundaries, as the support staff often discovering what learning tasks the teacher had scheduled in the classroom at the same time as the students. As there was no forewarning of planned activities by the teacher so there was some confusion for the support staff around how the teacher wanted certain tasks completed. All staff identified the lack of dedicated time to discuss and plan current/future activities, and to provide positive and/or critical feedback. They acknowledged however, that if this time were set aside it would address the lack of communication and potentially alleviate any confusion and guesswork between them.

Access to professional development training for some staff was raised as an issue. A number of support staff felt professionally excluded by not being invited to attend in-house training or attend weekly staff meetings. They also mentioned how they did not always receive relevant school-based information arising from staff meetings that had implications for their direct contact with students. The support and teaching staff were concerned about the restricted access to the files of high profile students placed in their classrooms, especially those that presented with violent tendencies and a history of expulsion from other schools. They discussed how this uncertainty undermined their sense of professional and personal confidence. In summary, the lack of communication between and amongst the staffing community in the school resulted in an on-going brewing sense of frustration from and towards each other, due to unmet expectations.

\subsection{Policies}

The term 'Support staff' was complex and confusing in the school as there were a total of five different types: an Aboriginal Education Officer, an Aboriginal Education Worker, an Aboriginal Education Resource Teacher, and two types of support staff that were funded separately and were responsible to work in class with students with special needs. The collective staff voice was unanimous that they were unable to accurately differentiate the numerous role and responsibility descriptors amongst themselves.

After recognising that confusion surrounding role descriptors and responsibilities was school-wide having been flagged by each participant as a significant issue, the training set out to clarify the different roles and responsibilities. The training cited the department's role descriptors for both teacher and support staff by explicitly highlighting the difference in duties and responsibilities for each role. Crucially, for both teachers and support staff desperately seeking the definitive 'line in the sand' that clarified role and responsibility division, the Department descriptors were still open to interpretation. The definition remained unclear by an end caveat in the support staff's role descriptor that they were required to "undertak[e] other related duties as determined by the Principal or the Supervisor" (Public Service Association, 2009). This caveat fuelled the blurry role definition between support staff and teacher that added to the confusion and uncertainty for the support staff. The confusion was particularly evident especially when the support staff moved between classes and worked with other teachers that demonstrated different interpretations of the support staff's role, or asked them to "undertake" what may have been deemed as a teaching role.

\subsection{Practice}

The training highlighted how the misunderstanding of both teacher and support staff expectations of each other in the classroom fuelled frustration, confusion and a lack of confidence between staff. Despite acknowledging this lack of clarity between roles, the teacher nor the support staff actively sought clarification or set boundaries with each other, so the situation remained unchanged only adding to the negative undercurrent of dissatisfaction. Ultimately, the support staff especially needed to be flexible and accommodate differences in teacher styles, attitudes and approaches to student management, and interpretation of their own support staff role. A support staff stated:

When I first started, it was really hard to know how to 'be' with certain teachers. I've finally worked it out. I know that when I am with [Teacher 1], I am not to verbally reprimand students because she wants to do all of that, but when I go to [Teacher 4's] class, she relies on me to be vocal and actively support her rules in the class with everyone (Support staff 3). 
Teachers also had their own viewpoints on the effectiveness of certain support staff allocated to their class:

The support staff I have in the afternoon is completely useless! Thank goodness for the one I have in the morning! She just gets in there, she knows what needs doing, keeps the students in-line if they are getting restless; she sees potential problems and acts on them before they get worse. The other one, well she just looks at me all the time constantly 'lost' in what to do next. I'm run ragged by the end of the afternoon because I might as well just have another student in the class (Teacher 9).

Despite the differences in tertiary qualifications and length of service, the research highlighted that teachers and the support staff voiced identical professional training needs. The training areas requested by both included: information on specific department's policies e.g. support staff /Teacher roles and responsibilities; 'Best Practice' teaching Indigenous students; and how to appropriately restrain students with special needs when required. Both cohorts sought information about general classroom behaviour management strategies, how to effectively manage a student crisis and anger, and managing students that display characteristics of Asperger Syndrome, Oppositional Defiance Disorder, and Conduct Disorder. Staff were also interested in how to best identify and manage students with mental health concerns e.g. Depression, Stress, Anxiety. Considering the nature of these training needs together with the blurred role boundaries that exist between the teachers and the support staff, the high level of staff dissatisfaction, turnover of staff, and stress reported in the school was understandable.

\section{Post-training Results}

The facilitator returned six weeks after the first professional development session. At this time school members reported either individually or as a group on the success of tried strategies and any benefits the clarity of role and responsibilities had made to their teaching practice and relationship with other staff. It became evident that three themes underpinned the teacher and support staff development: i) Communication; ii) Classroom practice and behaviour management; and iii) Planning.

\subsection{Communication}

Both the teachers and support staff agreed that there had been a much-welcomed increase in communication between the parties due to a conscious effort made by both to clarify boundaries. Clearer expectations of lesson structure content, roles and responsibilities in class, responding to student needs and boundary setting provided a greater sense of confidence for teacher and support staff. The introduction of staff pigeon holes provided a place where all staff, specifically casual and part-time staff, could be issued with meeting minutes and other relevant information pertaining to students. These staff were also extended the opportunity to attend training and development courses from which they had previously been excluded. Overall, the quality of relationships between all staff had improved markedly which was evident in the higher levels of staff morale.

\subsection{Classroom Practice and Behaviour Management}

The consequence of clearer and more frequent communication between teacher and support staff expectations of each other, translated in the classroom as more relaxed, confident, positive and inclusive lessons. The support staff reported having a more meaningful involvement in class as they worked cooperatively on programs and tasks with the teachers. They claimed to have greater confidence to participate in lessons evidenced by an increased feeling of being supported, taking initiative, and implementing reward systems. The support staff said that the improved classroom climate meant they could spend more time on their relationship with the students as a priority. Both teacher and support staff claimed they were more relaxed knowing what was required for class tasks, and felt an increase in the positive rapport with children.

\subsection{Planning}

One of the issues both teacher and support staff raised was the absence of planning between them despite spending for some, all day together. Both cohorts were encouraged to make and/or find the time i.e. one hour each week, to plan and discuss classroom tasks to see if it improved the current situation. Those who made time to communicate current and/or future classroom tasks with each other reported a greater sense of direction around classroom structure and execution of lessons. Some support staff claimed that it had felt as if their role had shifted from one of a 'child-minding service', to one where they were an integral part of a team in a cohesive classroom. Both teacher and support staff reported feeling 'on the same page' in the classroom, hence managing the content and student behaviours became more consistent and seamless.

\section{Discussion}

The SLIDE project demonstrates how an individualised approach to whole-school professional development training in schools effectively met the learning and training needs of teachers and support staff in a government 
primary school. The training was mindful to exercise all seven of the teacher-recognised factors for effective participation in professional development sessions (Borko et al., 2010; Kedzior \& Fifield, 2004; Little, 2006; Van Driel \& Berry, 2012).

From the outset, it was important that all members of the school community participated to ensure continuity in application of school practice. Significant to the success of the training can be found in the foundational design of the two sessions themselves. Each member of the school community was provided with a confidential and private 'space' to voice their concerns, needs and suggestions for improved practice. The design of the first session was to anonymously report the perceptions of the school community to each other, with resources and training suggestions for change. The second session provided staff with the opportunity for reflection, collaborative support, and follow-up. The training therefore was teacher-driven and had a clear focus to address individual and the broader needs of the school (Kedzior \& Fifield, 2004).

Central to the success of the training was the ability of each individual to self-reflect upon their practices from Pedagogical, Content, and Knowledge perspectives (Anderson \& Herr, 2011; Van Driel \& Berry, 2012). The facilitator needed to remind individuals to reflect upon and address their professional needs couched in the three dominant themes of communication, policy and practice emerging from the initial interview data.

The success and effectiveness of using the individual interview data as a collaborative voice for the basis of the whole-school training was assured for all participants as it addressed their self-assessed concerns. All staff had identified flaws and inefficiencies in their workplace, and given the opportunity to put forward positive suggestions in response. The facilitator shared these 'anonymous' suggestions with the school Principal for consideration, and all were integrated into the school day with the expectation that the whole-school would collectively move towards more effective and efficient working practices. Under the guise of a whole-school professional development day, all staff were able to anonymously raise practical and difficult interpersonal concerns to be addressed by a third person detached from the school community. In this way, no staff were singled out or viewed as not being as effective as others in the school, and the school community was given a 'clean sheet' to proceed with the daily running of the school.

The design of the two sessions with a six-week gap meant that the training was 'extended' and an action-research based model of inquiry and learning could take place based on the information shared in the first session (Little, 2006). Gathering as a group for the second session promoted a sense of coherence and integration of the information gathered and shared, and each person's experiences (Borko et al., 2010). Each individual's learning was promoted through inquiry and self-reflection through the active learning process (Van Driel \& Berry, 2012). Importantly, the onus of change for the better and the responsibility of personal and professional growth were laid directly upon each member of the school community in both sessions. Providing staff with the opportunity for self-evaluation and collaboration with their peers in the second session proved valuable in supporting each other's learning (Borko et al., 2010).

\section{Conclusion}

The success of the SLIDE project was a whole-school process that was collaborative and staff-driven. Identifying the different professional pressures and needs of all staff in the school community was pivotal to the design of training sessions that were meaningful and acted as a catalyst for positive change. Other important theoretical issues in management literature such as conflict management (Tekleab, Quigley \& Tesluk, 2009), would be a valuable inclusion in future related research projects. School executives planning forthcoming whole-school professional development days may consider how incorporating an individual focus may improve the overall meaningfulness and effectiveness of professional development training to the whole-school community.

\section{References}

Anderson, G., \& Herr, K. (2011). Scaling Up "Evidence-Based" Practices for Teachers is a Profitable but Discredited Paradigm, Educational Researcher, 6(40), 287-289.

Bielaczyc, K., \& Collins, A. (1999). Learning Communities in Classrooms: Advancing Knowledge for a Lifetime, NASSP Bulletin, 604(83), 4-10.

Bausmith, J., \& Barry, C. (2011). Revisiting professional learning communities to increase college readiness: The importance of pedagogical content knowledge. Educational Researcher, 40, 175-178. http://dx.doi.org/10.3102/0013189X11409927

Browder, D., Jimenez, B., Mims, P., Knight, V., Spooner, F., Lee, A., \& Flowers, C. (2012). The Effects of a "Tell-Show-Try-Apply" Professional Development Package on Teachers of Students With Severe 
Developmental Disabilities, Teacher Education and Special Education: The Journal of the Teacher Education Division of the Council for Exceptional Children, 3(35), 212-227.

Borko, H., Jacobs, J., \& Koellner, K. (2010). Contemporary approaches to teacher professional development. In Peterson P., Baker E., McGaw B. (Eds.), Third international Encyclopaedia of Education, 7, 548-556. Amsterdam, The Netherlands: Elsevier. http://dx.doi.org/10.1016/B978-0-08-044894-7.00654-0

Cuddapah, J., \& Clayton, C. (2011). Using Wenger's Communities of Practice to Explore a New Teacher Cohort, Journal of Teacher Education, 62(1), 62-75.

Friere, P. (1970). Pedagogy of the Oppressed. London: Penguin.

Guskey, T. (2003). Analyzing lists of the characteristics of effective professional development to promote visionary leadership. NASP Bulletin, 87(637), 38-54.

Kedzior, M., \& Fifield, S. (2004). Teacher Professional Development, Education Policy Brief, Education Research and Policy Centre, University of Delaware, 15, 1-6.

Little, J. (2006). Professional community and professional development in the learning-centered school. Arlington, VA: National Education Association.

Opfer, D., \& Pedder, D. (2011). Conceptualizing Teacher Professional Learning. Review of Educational Research, 81(3), 376-407.

Public Service Association of NSW. (2009). School Learning Support Officer, http://psa.labor.net.au/schools/1252900732_28578.html, Retrieved 14 April 2010.

Renwick, R., Fudge-Schormans, A., \& Zekovic, B. (2003). Quality of life for children with developmental disabilities. Journal on Developmental Disabilities, 10, 107-114.

Rhoton \& Stile, (2002) Exploring the professional development design process: Bringing an abstract framework into practice. Science Educator, 11(1), 1-8. http://dx.doi.org/10.1023/A:1013048828150

Tekleab,A., Quigle, N., \& Tesluk, P. (2009). A Longitudinal Study of Team Conflict, Conflict Management, Cohesion, and Team Effectiveness. Group Organisation Management, 34(2), 170-205. http://dx.doi.org/10.1177/1059601108331218

Van Driel, J., \& Berry, A. (2012). Teacher Professional Development Focusing on Pedagogical Content Knowledge. Educational Researcher, 41(1), 26-28.

Vrasidas, C., \& Glass. G. (2004). Online Professional Development for Teachers, Information Age Publishing: New York.

Webster-Wright, A. (2009). Reframing Professional Development Through Understanding Authentic Professional Learning. Review of Educational Research, 79(2), 702-739.

Wenger, E. (1998). Communities of Practice, Learning, Meaning and Identity, Cambridge, UK: Cambridge University Press.

\section{(cc) $\mathrm{Br}$}

This work is licensed under a Creative Commons Attribution 3.0 License. 